\title{
Economic evaluation of seizures associated with solitary cysticercus granuloma
}

\author{
J. M. K. Murthy, G. Rajshekar* \\ Department of Neurology, The Institute of Neurological Sciences, CARE Hospital, Hyderabad and *Department of Neurology, \\ Apollo Hospital, Hyderabad, India
}

Background: Patients with solitary cysticercus granuloma (SCG) develop acute symptomatic seizures because of the inflammatory response of the brain and the seizures are self-limiting. Thus seizure disorder associated with SCG provides a good model to study the total cost of illness (COI). Materials and Methods: COI of new-onset seizures associated with SCG was studied in 59 consecutive patients registered at the epilepsy clinic. Direct treatmentrelated costs and indirect costs, man-days lost and wages lost were evaluated. The relative cost was calculated as the percentage of per capita gross national product (GNP) at current prices for the year 1997-1998. Results: The total COI, for treating seizure disorder associated with SCG per the period of CT resolution of the lesion per patient was INR 7273.7 (US\$ 174.66, I\$ 943.16) and he/she would be spending $50.9 \%$ of per capita GNP The direct cost per patient was INR 5916 (US\$ 137.14, 41.4\% of per capita GNP). If the patient had received only AEDs for the period of resolution of CT lesion, the cost would be INR 5702.48 (US\$132.2, 40\% of per capita GNP). The extra expenditure on albendazole and steroid was INR 213.72 (US\$ 4.95), $3.6 \%$ of the total direct cost and $20.7 \%$ of the medication cost. Indirect cost (average wage loss) per patient was INR 1312.7 (US\$ 30.42) and it accounted for $9 \%$ of per capita GNP. The one-time expenditure at present costs (adjusted for inflation) to the nation to treat all the prevalence cases is to the tune of INR 1.184 billion (US\$2.605) and $0.0037 \%$ of GNP. Conclusions: This study suggests that seizure disorder associated with SCG, a potentially preventable disorder, is a good model to study the total COI. The one-time expenditure at present costs to the nation to treat all the prevalence cases of seizure disorder associated with SCG is to the tune of INR 1.184 billion (US\$ 2.605 million) and $0.0037 \%$ of GNP.

Key words: Cost of illness, direct cost, indirect cost, seizures, solitary cysticercus granuloma
Neurocysticercosis (NCG) is a major public health problem in developing countries and seizures are the most frequent clinical manifestation. Seizures occur with all the evolutionary stages. ${ }^{[1]}$ Single computerized tomography (CT)-enhancing lesion, measuring less than $20 \mathrm{~mm}$, commonly seen in patients with seizures in countries endemic to NCC represents solitary cysticercus granuloma (SCG). ${ }^{[2,3]}$

Even though overabundance of reports of this CT lesion came from India, these lesions have been reported from all over the world. ${ }^{[4]}$ People with SCG develop acute symptomatic seizures because of the inflammatory response of the brain..$^{[3,5]}$ Recent long-term follow-up studies have shown that seizures associated with SCG have good prognosis and antiepileptic drugs (AEDs) can safely be withdrawn with the resolution CT lesion. ${ }^{[5-7]}$ Thus SCG provides a good model to study the total cost of illness.

\section{Materials and Methods}

Cost of illness (COI) of seizure disorder associated with SCG was studied in 59 consecutive patients registered between January 1997 and December 1997 in the epilepsy clinic of a state university hospital located in the capital city of Andhra Pradesh, a province in south India. We included only patients with new-onset seizures who had not received any specific treatment or AEDs. All patients were treated with AED monotherapy (phenytoin or carbamazepine) for the period of CT resolution of the lesion. Drug dose was based on the body weight and clinical response and no drug monitoring was done. They also received a course of albendazole $(15 \mathrm{mg} / \mathrm{kg}$ per day for four weeks) and steroids (1-2 mg/kg per day for four weeks with tapering dose). All patients were treated in the outpatient clinic. AED withdrawal was gradual over a period of two months. Patients were advised not to come for further follow-up after stopping AEDs. 


\section{Estimation of direct costs}

For direct costs' analysis of those costs required to manage the illness, direct treatment-related costs, after registration in the epilepsy clinic were considered. These included costs of medications, doctor contacts at all levels, investigations (EEG and CT scan) and nonmedical costs (transportation). For investigations the tariffs of the hospital (Indian National Rupee, INR) at the time of data collection were considered [Table 1]. While calculating the transportation costs the actuals spent by the patient and accompanying persons from their place of living, including transportation from bus or railway station to our institute were considered. The cost of AEDs was calculated using the prices for the brand-name products in INR. These prices included basic net and local taxes. All patients had one interictal EEG and brain CT at the time of registration. First repeat CT scan was done after six months of the index scan. This time interval for the first scan was based on the data that a greater proportion of lesions would resolve partially or completely by six months and repeat CT examinations could be avoided in a substantial proportion (67\%) of patients. ${ }^{[8]}$ In patients in whom CT scan showed persistence of the lesion, CT was repeated at three-month intervals till the lesion disappeared. Patients in whom the lesion got calcified were excluded from the analysis because some of these patients may develop remote symptomatic epilepsy. ${ }^{[6,7,9]}$

\section{Estimation of indirect costs}

Productivity loss was measured by the number of lost man-days due to recurrence of seizures and also due to travel for follow-up visit. In the case of students, productivity loss was measured by the number of days they abstained from school or college. While calculating wage losses for the daily wage earners, the actual wages were calculated. For people who were employed the actual losses were calculated. For students only man-days lost were considered.

\section{Cost calculation}

The cost per person was computed as the mean cost for the whole cohort. The relative cost was calculated as the

Table 1: Unit cost of investigations, antiepileptic drugs, and transportation

\begin{tabular}{lcc}
\hline & \multicolumn{2}{c}{ Unit cost } \\
\cline { 2 - 3 } & INR & US\$ \\
1. Investigations EEG & 200 & 4.63 \\
CT scan & 1800 & 41.72 \\
2. antiepileptic drug (PDD/DDD) & 8.95 & 0.20 \\
Carbamazepine phenytoin & 2.73 & 0.06 \\
phenobarbitone & 1.89 & 0.04 \\
3. Transportation & 1.75 & 0.04 \\
**Passenger bus & & \\
Express bus & 2.85 & 0.06 \\
\hline
\end{tabular}

${ }^{*}$ Conversion rate: One US $\$=43.14 \mathrm{INR},{ }^{* *}$ This represents cost for unit (1 kilometers ); while calculating, the actual money spent by the patients and accompanying persons was taken into consideration percentage of gross national product (GNP) at current prices for the year 1997-98, INR 137694.3 billion (US\$ 31918 billion) and per capita GNP at current prices INR 14,284 (US\$ 296.72). ${ }^{[10]}$ To make international cost comparisons, costs in US dollar (US\$) and International Dollars (I\$) were also calculated. The calculation is obtained by adjusting for the purchasing power parity (PPP) factor 5.4. ${ }^{[11]}$

\section{Results}

The demographic data was: mean age: 18.11 years (range 11-26); male to female distribution: $35: 24$; the mean number of seizures before registration: 3.95 (range 1-6) and the total duration of illness 11.71 months (range 114). Twelve patients (20\%) had three CT scans and three (5\%) had four CT scans before AED withdrawal. The treatment protocol was safe and effective [Table 2]. No patient required hospitalization with this treatment protocol.

The direct cost per patient treated with AEDs for the period of resolution of CT lesion and a course of albendazole and steroids was INR 5916 (US\$ 137.14; $41.4 \%$ of per capita GNP). If the patient had received only AEDs for the period of resolution of CT lesion, the cost would be INR 5702.48 (US\$132.2; 40\% of per capita GNP). A patient would be spending 3.6\% (INR 213.7, US\$ 4.95, $1.5 \%$ of per capita GNP) of the total direct cost on albendazole and steroids and it accounted for $20.7 \%$ of total medication costs [Table 3].

The number of days lost due to seizures for the total cohort after starting treatment was 32 days. Days lost due to travel for follow-up visits for the entire cohort were 170 days. This included 65 student days lost. It was 162 days for the parents and/or relatives. Total wage loss was

Table 2: Outcome measures

Mean frequency of seizures

In patients with $<6$ months duration $\quad 0.87$

In patients with $>6$ months duration $\quad 0.23$

Albendazole and steroid-related adverse Nil

drug reactions

Significant AED-related adverse events $\quad 10 \%$

Seizure relapse after AED withdrawal Nil

Mean duration of follow-up after AED 22-6

Withdrawal (months)

AED - Antiepileptic drugs

Table 3: Cost of Illness - direct costs per patient

INR US\$

Outpatient medical consultations Investigations

Travel and other expenditure

AED and other medication

Total cost

Costs in International Dollar (PPP)

Per capita GNP (at current prices)

$125.42 \pm 29.00$

2.91

$4166.10 \pm 730.76 \quad 96.57$

$592.78 \pm 701.61 \quad 13.74$

$1031.90 \pm 123.81 \quad 23.92$

$5916.20 \pm 1756.66 \quad 137.14$

740.56

Per capita GNP (at current prices) for the year US\$ 450, PPP - Purchasing power parity, AED - Antiepileptic drugs 
INR 77450 (US\$ 1801.2). Indirect cost (wage loss) per patient was INR 1312.7 (US\$ 30.42, 9\% of per capita GNP) [Table 4].

The total COI for treating seizure disorder associated with SCG for the period of resolution of CT lesion per patient was INR 7273.7 (US\$ 174.66; I\$ 943.16) and he/ she would be spending $50.9 \%$ of per capita GNP.

In our community-based Comprehensive Rural Epilepsy Study South India (CRESSI), the prevalence of seizure disorder associated with SCG was 0.10 per 1000 population (unpublished data), this was the only such study in the country. Extrapolating these figures to the present population of India (1.107 billion - 2005-2006) ${ }^{[10]}$ the number prevalence cases of seizure disorder associated with SCG in India would be 110,700. After adjusting for the inflation, the projected one-time expenditure to the nation to treat all the prevalence cases is to the tune of INR 1.184 billion (US $\$ 2.605$ million), which is $0.003 \%$ of total GNP (GNP at current prices 2005-2006 INR 31,829. 49 billion). ${ }^{[10]}$

\section{Discussion}

The current environment of cost consciousness in healthcare has resulted in a need for the economic evaluation of different treatments in order to identify those which provide the most value for money in treating patients. This has much more relevance for developing countries. In developing countries the per capita expenditure on healthcare is very minuscule and in India the expenditure is only US $\$ 21$ and $78 \%$ of the cost of healthcare is paid by patients. ${ }^{[12]}$ The results of the present economic evaluation indicate that under base-case assumptions patients with seizures associated with SCG need AED therapy for the period of resolution of CT lesion, this approach is safe and effective. However, the patient will be spending a considerable proportion of his/her per capita GNP, that is $50.9 \%$.

The treatment of seizures associated with SCG is highly variable. Most clinicians treat these patients with AEDs for a two-three year seizure-free period, just like as they would treat any other type of epilepsy. ${ }^{[13]}$ Long-term follow-up studies of patients with SCG suggest that seizures associated with SCG should be treated with AEDs

\section{Table 4: Cost of Illness indirect costs}

\begin{tabular}{|c|c|}
\hline \multicolumn{2}{|c|}{ Number of man-days lost (productivity loss) } \\
\hline Days lost due to seizures & 32 days $(0-2)$ \\
\hline Days lost due to travel & \\
\hline Patient's & 170 days* $(1-2)$ \\
\hline Relatives'/parents' & 162 days $(1-2)$ \\
\hline${ }^{*}$ (Student days loss-65 days) & \\
\hline Total wage loss & $\begin{array}{c}\text { INR: } 77450.00 \\
\text { (US } \$: 801.2 ; \text { IN } \$: 9726.48)\end{array}$ \\
\hline Average wage loss per patient & INR: $1312.70(180-2450)$ \\
\hline $\begin{array}{l}\text { Per capita GNP } \\
\text { (at current prices) }\end{array}$ & $9.2 \%$ \\
\hline
\end{tabular}

Per capita GNP (at current prices) for the year 1997-98 INR 14,284 for two reasons: (i) seizures are likely to recur as long as the lesion persists and (ii) the time period for the resolution of the CT lesion is quite variable. There is abundant evidence to suggest that AEDs can safely be withdrawn once the follow-up scan shows resolution of the lesion. ${ }^{[14]}$ The place of cysticidal therapy in patients with SCG is not clear. The argument against cysticidal therapy is that seizures associated with this lesion are because of the inflammatory response of the brain and the lesions are known to resolve spontaneously. ${ }^{[1]}$ However, the results of the meta-analysis of the cysticidal drug trials in the treatment of NCC suggests that in patients with enhancing lesions, the risk for seizure recurrence is lower after cysticidal treatment and also trend toward lesion resolution. ${ }^{[15]}$ Based on these findings our approach to treat these patients with a course of cysticidal therapy and AEDs for the period of resolution of CT lesion has a good rationale. In this study the additional cost for this added therapy accounted for $3.6 \%$ of the total COI.

Neurocysticercosis is of great economic relevance, resulting from the cost of medical treatment, lost working days and losses due to livestock condemnation. ${ }^{[16]}$ In developing countries seizure disorder associated with NCC represents a significant proportion of the treatable burden of disease. But the developing world with population five times the size of the developed world has at its disposal only $25 \%$ of the global total gross domestic product (GDP) and 5\% of the global per capita GDP ${ }^{[16-19]} \mathrm{A}$ minimum estimate of the cost of admission to hospital and wage loss for NCC in the United States (a nonendemic country) was US\$ 8.8 million annually, whereas estimated treatment costs in Mexico were US\$ 89 million and in Brazil US\$ 85 million. ${ }^{[20]}$ From this study one-time expenditure (after adjusting for the inflation) to the nation to treat all the prevalence cases of seizure disorder associated with SCG in India is US\$ 2.605 million (0.0038\% of total GNP at current prices). There are no incidence studies to project the likely annual costs to treat the seizure disorder associated with this lesion. This economic burden is avoidable to a significant extent with well-designed public health measures and public health education as has been done in Reunion Island. ${ }^{[21]}$

The principal aim of COI is to measure the economic burden of illness to the society. The COI can provide information to support the political process as well as the management functions at different levels of the healthcare organization. ${ }^{[22]}$ However, its usefulness as a policy decision-making tool has been questioned since its inception. ${ }^{[21-24]}$ Other forms of economic evaluation (e.g., cost-effectiveness, cost-benefit analysis) would be much more useful for those taking decisions and ranking priorities. ${ }^{[21,25]}$ However, COI evaluation of totally preventable diseases, like this one with considerable economic burden to the nation provides useful information for policymakers for effective preventive measures that 
will be cost-saving and cost-effective.

When considering the results of this economic evaluation the limitations of the study must be recognized. The costs involved reflect the protocol followed in this study and this treatment strategy has a sound scientific basis as discussed above. As regards cysticidal drug and steroid therapy, this is not a randomized control study. While evaluating indirect costs only man-days loss and wage loss were considered.

\section{References}

1. Carpio A, Escobar A, Hauser WA. Cysticercosis and epilepsy: A clinical review. Epilepsia 1998;39:1025-40.

2. Rajshekhar V, Chacko G, Haran RP, Chandy MJ, Chandi SM. Clinicoradiological and pathological correlations in patients with solitary cysticercus granuloma and epilepsy: Focus on presence of the parasite and edema formation. J Neurol Neurosurg Psychiatry $1995 ; 59: 284-6$.

3. Del Brutto OH, Rajashekhar V, Whit AC Jr, Tsang VC, Nash TE, Takayanagui OM, et al. Proposed diagnostic criteria for neurocysticercosis. Neurology 2001;57:177-83.

4. Rajshekhar V, Chandy MJ. Incidence of solitary cysticercus granulomas. In: Rajashekhar V, Chandy MJ, editors. Solitary cysticercus granuloma. Chennai Oriental Longman Ltd: 2000a. p. $12-28$.

5. Murthy JM, Reddy YV. Prognosis of epilepsy associated with single CT enhancing lesion: A long-term follow-up study. J Neurol Sci 1998;159:151-5

6. Carpio A, Hauser WA. Prognosis for seizures recurrence in patients with newly diagnosed neurocysticercosis. Neurology 2002;59:1730-4.

7. Rajshekhar V, Jeyaseelan L. Seizure outcome in patients with a solitary cerebral cysticercus granuloma. Neurology 2004;62:2236-40.

8. Rajshekhar V. Rate of spontaneous resolution of a solitary cysticercus granuloma in patients with seizures. Neurology $2001 ; 57: 2315-7$

9. Murthy JM, Reddy VS. Clinical characteristics, seizure spread patterns and prognosis of seizures associated with a single small cerebral calcific CT lesion. Seizures 1998;7:153-7.

10. Available from: http://rbidocs.rbi.org.in/rdocs/Publications/PDFs/ 72631.pdf.

11. Statistical outline of India: 2002-2003, Department of Economics and Statistics. Tata Services Limited: Mumbai; 2003.

12. Murray CJ, Govindraj R, Musgrove P. National health expenditures: A global analysis. In: Murray CJL, Lopez AD, editors. Global comparative assessments in the health sector: Disease burden, expenditure and interventional package. World Health Organization: 1994. p. 141-55

13. Rajshekhar V, Chandy MJ. Medical management of solitary cysticercus granuloma. In: Rajashekhar V, Chandy MJ, ediors. Solitary cysticercus granuloma. Chennai Oriental Longman Ltd: 2000 c. p. 112-34.

14. Murthy JM. Seizures associated with solitary cysticercus granuloma: Antiepileptic drugs for how long? Neurol India 2006;54:141-2.

15. Del Brutto OH, Roos KL, Coffey CS, Garcia HH. Meta-analysis: Cysticidal drugs for neurocysticercosis: Albendazole and praziquantel. Ann Inern Med 2006;145:43-51.

16. Pal DK, Carpio A, Sander JW. Neurocysticercosis and epilepsy in developing countries. J Neurol Neurosurg Psychiatry $2000 ; 68: 137-43$.

17. The World Bank, Entering the $21^{\text {st }}$ century. World Development Report: 1999/2000. Oxford University Press: New York; 2000.

18. United Nations, Handbook of international trade and development statisties, 1996-1997. United Nations: New York; 1999.

19. International Monitory Fund, Balance of payments statisties: Yearbook, Part 2: world and regional tables 1998. International Monitory Fund, Publication Services: Washington DC; 1995.

20. Roberts T, Murrell KD, Marks S. Economic losses caused by food borne parasitic diseases. Parasitol Today 1994;11:419-23.

21. Mignard D, de Lacroix-Mignard CM. Neurocysticercosis and epilepsy: Large improvement with a better environment. Epilepsia 1999;40:192.

22. Tarricone R. Cost-of-illness analysis: What room in health economies? Health Policy 2006;77:61-3.

23. Shiell A, Gerad K, Donaldson C. Cost of illness studies: An aid to decision making? Health Policy 1987;8:317-23.

24. Koopmanschap MA. Cost-of-illness studies: Useful for health policy? Pharmacoeconomics 1998;14:143-8.

25. Byford S, Torgerson DJ, Raftery J. Economic note: Cost of illness studies. BMJ 2000;320:1335

Accepted on 14-11-2006

Source of Support: Nil, Conflict of Interest: None declared. 